\title{
Genotypic and Organ Variation in Ginsenoside Contents from American Ginseng Populations
}

\author{
Jinwook Lee ${ }^{1}$ \\ Department of Integrative Plant Science, Chung-Ang University, Anseong 17546, Republic of Korea \\ Kenneth W. Mudge \\ School of Plant Science, Horticulture Section, 13 Plant Science Building, Cornell University, Ithaca, \\ NY 14853
}

\begin{abstract}
Additional Index words. Panax quinquefolius, root and leaf ginsenosides, estimated root age, principal component analysis, hierarchical cluster analysis

Aвstract. Variation in ginsenoside content was investigated as a function of population/genotype, plant organ, and age using four geographically isolated wild populations and one landrace population of american ginseng (Panax quinquefolius L.). The contents of individual and total ginsenosides were affected by the main and two-way interactions between population, organ, and age. Ginsenoside Re was not detected in roots of the wild population plants but was found in leaves and in both organs of the landrace population. A positive relationship between root age and total root ginsenosides was detected in two wild populations. Individual root ginsenosides were highly correlated with certain leaf ginsenosides in wild populations rather than in landrace populations. Therefore, the results suggest that certain leaf ginsenosides would be applied for potential biomarkers to estimate individual root ginsenosides. Principal component analysis (PCA) scores plot indicates that all wild populations were segregated from the single landrace population. However, cluster analysis indicates that differences existed between organs, and between the wild and landrace populations. Overall, the result suggests that the variation of individual and total ginsenoside contents would be influenced by a combination of population, plant organ, and root age.
\end{abstract}

American ginseng contains the secondary active pharmaceutical compounds, triterpene saponins, known as ginsenosides (Attele et al., 1999; Li, 1995; Proctor and Bailey, 1987). More than 30 kinds of ginsenosides have been isolated and structures reported in ginseng (Chuang and Sheu, 1994; Luchtefeld et al., 2004; Mahady et al., 2000). Individual or total ginsenosides have one or more specific beneficial functions and effects on human physiology and health, such as antitumor, antistress, central nervous system stimulation, and adaptogenic effects (Attele et al., 1999; Mahady et al., 2000; Qi et al., 2011; Sivakumar et al., 2005).

Ginsenosides have been classified into three categories, panaxadiols, panaxtriols, and oleanic acid, based on molecular structure, especially type and number of sugar moieties, and number and site of hydroxyl group (Attele et al., 1999). In american ginseng, the two major groups are 20(S)-protopanaxadiol and 20(S)-protopanaxatriol (Li et al., 1996; Wang et al., 2005, 2006). The most abundant ginsenosides reported in american ginseng root are ginsenosides $\mathrm{Rg} 1$ and Re, belonging to 20(S)protopanaxatriol, and ginsenosides $\mathrm{Rb} 1, \mathrm{Rc}, \mathrm{Rb} 2$, and $\mathrm{Rd}$, belonging to 20(S)-protopanaxadiol (Corbit et al., 2005; Qi et al., 2011). Ginsenosides $\operatorname{Rg} 1, \operatorname{Re}$, and Rb1 are higher than ginsenosides $\mathrm{Rc}, \mathrm{Rb} 2$, and $\mathrm{Rd}$ in wild american ginseng populations (Assinewe et al., 2003; Lim et al., 2005; Schlag and McIntosh, 2006). However, the composition and contents of root ginsenosides in wild ginseng populations varies greatly (Assinewe et al., 2003; Lim et al., 2005), compared with landrace populations with respect to cultivation method,

Received for publication 23 Apr. 2018. Accepted for publication 30 May 2018. We gratefully acknowledge the technical support of Joe Lardner and Wansang Lim from the School of Integrative Plant Science, Horticulture Section, Cornell University.

${ }^{1}$ Corresponding author. E-mail: j1425@cau.ac.kr. season, and location (Chung et al., 2012; Lee et al., 2011; Lim et al., 2005; Smith et al., 1996). Even within a single ginseng farm, the contents of ginsenosides varied greatly (Hong et al., 2005). The storage root of american ginseng is the only organ that is used widely and commercially to meet the high demand for the consumption of pharmaceutical products and health supplements, but all the organs of american ginseng contain ginsenosides (Konsler and Shelton, 1990). The contents and composition of individual ginsenosides vary highly among ginseng organs (Jackson et al., 2003; Kim and Choi, 1987; Konsler and Shelton, 1990; Li and Mazza, 1999; Li et al., 1996; Wang et al., 2006). Among roots of individual plants, there is significant variation in the compositions and contents of individual and total ginsenosides (Smith et al., 1996). Similarly, among the roots of 6-year-old asian ginseng (Panax ginseng Meyer) plants, there was variation in the contents of the individual ginsenosides (Nam et al., 1998). Furthermore, the contents and compositions of ginsenosides in root are strongly affected by seasonal variation (An et al., 2002; Jochum et al., 2007; Kim et al., 1981; Li and Wardle, 2002), storage root age (Court et al., 1996; Mudge et al., 2004; Soldati and Tanaka, 1984), soil fertility (Konsler and Shelton, 1990), soil nutrients (Lee and Mudge, 2013a), and water deficit (Lee and Mudge, 2013b). Leaf ginsenosides are also influenced by the maturity of the foliar organ ( $\mathrm{Li}$ et al., 1996). The contents of six major ginsenosides and total ginsenoside show considerable genotypic variation among geographically isolated wild ginseng populations (Assinewe et al., 2003; Konsler et al., 1990; Lim et al., 2005; Mudge et al., 2004). Also, the compositions of ginsenoside differ between root and foliar organs within the landrace american ginseng populations ( $\mathrm{Li}$ and Mazza, 1999; Li et al., 1996). In addition, the contents of root ginsenosides were highly correlated with the estimated ginseng root age 
(Court et al., 1996; Mudge et al., 2004; Qu et al., 2009; Shi et al., 2007; Soldati and Tanaka, 1984), although not in all cases (Qu et al., 2009; Shi et al., 2007).

Ginsenoside Re and total ginsenosides in leaves may serve as phytochemical biomarkers for root ginsenosides because the accumulation pattern of ginsenoside $\mathrm{Re}$ and total ginsenoside in root was closely associated with those in leaf (Assinewe et al., 2003; Wang et al., 2006). Therefore, the primary objective of this study was to test the hypothesis that the contents of particular ginsenosides in leaves might be linked with the corresponding ginsenosides, the other ginsenosides in the roots from wild and landrace american ginseng populations or both. Additional objectives were to determine the contents and compositions of leaf and root ginsenosides between geographically isolated wild and landrace populations, to elucidate the effect of a storage root age on ginsenoside accumulation from wild ginseng populations. An additional objective was to evaluate the usefulness of non- or less destructively harvested leaf ginsenosides as biomarkers for root ginsenosides for screening for high quality ginseng population among geographically isolated wild populations of american ginseng.

\section{Materials and Methods}

SAMPle COllection AND PREPARATION. Several geographically isolated wild populations of american ginseng were identified and individual plants were tagged. The selected wild populations were growing under the dense shade of deciduous hardwood trees on northwest-, north-, northeast-, or east-facing slopes within Cornell University's Arnot Teaching and Research Forest (ATRF), near Van Etten, NY (Chemung County). Among the selected populations, four populations were identified. Each population was physically separated from all other populations by at least $1.5 \mathrm{~km}$ distance. The location of each population was recorded with a global positioning system. Soil samples from $10 \mathrm{~cm}$ depth of soil from soil surface were taken within $30 \mathrm{~cm}$ distance from the identified wild ginseng plants on 8-11 Aug. Ten soil samples per population were pooled and were then air-dried for $7 \mathrm{~d}$. Nutrient elements were analyzed by inductively coupled plasma spectrophotometry at the Plant and Soil Nutrient Analysis Laboratory, Department of Crop and Soil Sciences, Cornell University, Ithaca, NY. Twelve individual ginseng plants, which had three prongs and five leaflets per prong, for total of 15 leaflets were randomly taken from each wild ginseng population and then shoot growth characteristics were evaluated. The roots were wrapped with a damp paper towel, put into a ziplock plastic bag, transferred to the laboratory, and then stored at $4{ }^{\circ} \mathrm{C}$ on 15 and 17 Aug. Twelve landrace ginseng plants, which originated from Wisconsin and seeded in 2000 at the forest garden of Cornell University's ATRF were taken on 17 Aug. After transfer to the laboratory, ginseng plants were washed with tap water to remove soil and then blotted with a paper towel to remove remaining water. After completing the measurement of shoot and root fresh weight $(\mathrm{FW})$, leaf areas were recorded with a leaf area meter (LI-3100; LI-COR, Lincoln, NE) to obtain leaf area index (LAI). The age of each ginseng plant was estimated by counting the annual bud scars along the rhizome (Lim, 2005). Root diameter and total root length were also measured. Shoot tissues were dried at $70{ }^{\circ} \mathrm{C}$ for $3 \mathrm{~d}$, for the measurement of shoot dry weight (DW) and analysis of leaf ginsenosides. Roots were placed in a forced-air food dehydrator at $35^{\circ} \mathrm{C}$ for $7 \mathrm{~d}$ for the measurement of root DW and the analysis of root ginsenosides. Dried ginseng roots and leaves were prepared by grinding to a powder with a tissue grinder (AG-2005; Angel Electronic, Seoul, Korea) and passed through a 60-mesh sieve screen for the analysis of ginsenosides.

GinsenOSIDE EXTRACTION AND ANALYSIS. Ginsenoside analysis followed the procedure of Lee and Mudge (2013a). One hundred milligram dried ground ginseng leaf and root tissue was extracted in $30 \mathrm{~mL}$ of $70 \%$ methanol $(\mathrm{MeOH})$. The $\mathrm{MeOH}$ extract was vacuum-evaporated at $38{ }^{\circ} \mathrm{C}$ with a rotary evaporator (011; BUCHI Analytical, New Castle, DE), redissolved in $5 \mathrm{~mL}$ of $100 \% \mathrm{MeOH}$, and dried with a rotary evaporator. The residue was redissolved in $500 \mu \mathrm{L}$ of $16 \%$ acetonitrile. Samples of $15 \mu \mathrm{L}$ were analyzed by high-performance liquid chromatography [HPLC (2690 Separations Module HPLC; Waters Corporation, Milford, MA)], with a photodiode array detector (996, Waters) as an ultraviolet detector at $203 \mathrm{~nm}$. Empower Pro software (Build 1154, Waters) was used for solvent gradient, peak identification, and integration. A reversed phase $\mathrm{C}_{18}$ column [packing material LiChrosorb RP18, particle size $5 \mu \mathrm{m}$, length $250 \mathrm{~mm}$, internal diameter $3 \mathrm{~mm}$ (HPLC Column; Varian, Lake Forest, CA)] was used with a guard column (Reversed Phase ChromSep Guard Column SS; Varian). A composition gradient of the two eluents of the mobile phase (A) $0.14 \%$ phosphate buffer and (B) $100 \%$ acetonitrile was $0-20$ $\min , 84 \%$ to $82 \% \mathrm{~A}, 16 \%$ to $18 \% \mathrm{~B} ; 20-60 \mathrm{~min}, 82 \%$ to $60 \% \mathrm{~A}$, and $18 \%$ to $40 \% \mathrm{~B}$. The flow rate was $1.15 \mathrm{~mL} \cdot \mathrm{min}^{-1}$. As an internal standard, $m$-cresol (Sigma-Aldrich Chemical Co., St. Louis, MO) was added into each sample to confirm the injection volume and retention time per injection. Ginsenoside standards included ginsenosides Rg1, Re, Rb1, Rc, Rb2, and Rd (Indofine Chemical Co., Hillsborough, NJ). Individual ginsenosides from the extracts were identified and quantified by retention time and peak areas as compared with those of authentic ginsenoside standards.

Statistical analysis. Each dependent variable (plant growth characteristics, and leaf and root ginsenosides) was individually analyzed using analysis of variance [ANOVA (SAS version 8.02; SAS Institute, Cary, NC)]. Following analysis of variation, mean separations of plant growth characteristics, and leaf and root ginsenosides were performed using Duncan's multiple range tests at $P=0.05$ based on the mean square error for each dependent variable. Linear regression analysis was performed to determine the relationship between total ginsenosides and estimated storage root age by using the SigmaPlot (version 10.0; Systat Software, San Jose, CA). Pearson correlation coefficient analysis was performed to determine the relationships between plant growth characteristics and root and leaf ginsenosides. Pearson correlation coefficients were obtained by subjecting the data set using the correlation coefficient procedure (PROC CORR) in SAS (version 8.02). Red and blue colors indicated a positive and negative correlation coefficient between factors. To test whether leaf ginsenoside could contribute to the accumulation of root ginsenoside, analysis of covariance (ANCOVA) was performed using ANCOVA procedure (PROC ANCOVA) by controlling leaf ginsenosides.

To provide an overview of population diversity on the contents of ginsenosides, a PCA was applied to visualize population diversity and segregation between ginseng populations in ginseng organs based on the composition and contents of ginsenosides. The mean values of each ginsenoside from all 
populations were transformed by mean centering and SD scaling and then PCA was performed using The Unscrambler (version 10.0.1; Camo, Trondheim, Norway). For the validation test, random cross validation method was applied by using 20 segments with three samples per segment. In addition, for the evaluation of phylogenetic relationships, the transformed data set was also used for hierarchical cluster analysis by using The Unscrambler (version 10.0.1), with hierarchical complete linkage clustering method and squared Euclidean as distance measure.

\section{Results}

Soil nutrients were mostly greater in wild populations than in landrace population. However, soil S, Fe, Mn, and Al contents were higher in landrace population than in wild populations. Soil acidity was about one unit lower in wild populations than in landrace population, whereas the organic matter was $\approx 1.5 \%$ higher in wild populations than in the landrace population (Table 1).

Of plant growth characteristics, shoot DW, root diameter, root FW and DW, and estimated ginseng root age were higher in wild populations than in landrace population. However, sympodium height (stalk length from soil surface to base of petiole), leaf area, shoot FW, and total root length were not different among wild and landrace populations (Table 2).

Correlation analysis indicated that all plant growth parameters were positively correlated with each other but that leaflet number was not significantly correlated with any other plant growth parameters in wild populations (Fig. 2A). By contrast, certain plant growth parameters, i.e., plant height, root length, root diameter, and leaf number were negatively correlated in landrace population (Fig. 2B).

The compositions and contents of six individual and total ginsenosides from wild and landrace populations showed differences between leaf and root (Table 3). In leaves, individual and total ginsenosides showed a significant variation among populations except for ginsenoside Rb1. Leaf ginsenosides Re, $\mathrm{Rd}$, and total ginsenoside were greater in landrace population than in wild populations. Root ginsenosides Rg1 and Rb1 were higher in wild populations than in landrace population but root ginsenosides $\mathrm{Re}$ and $\mathrm{Rd}$ were lower in wild populations. Intriguingly, in roots, ginsenoside Re was not detected in any wild populations but present only in landrace population. As a main effect, the contents of ginsenosides Rc, Rb2, Rd, and total were affected by population. All ginsenosides were affected by organ (leaf and root) but only ginsenosides Rg1, $\mathrm{Rb} 1$, and total were affected by the estimated storage root age. There were significant two-way interactions between ginsenosides $\mathrm{Rg} 1$ and $\mathrm{Rb} 2$ for population and organs $(\mathrm{P} \times \mathrm{O})$, ginsenoside $\mathrm{Rc}$ and total ginsenoside for ginseng population and root age $(\mathrm{P} \times \mathrm{A})$, and ginsenosides $\mathrm{Rg} 1, \mathrm{Rb} 1, \mathrm{Rc}$, and total ginsenoside for root age and organs $(\mathrm{A} \times \mathrm{O})$. There was no statistically significant three-way interaction.

To compare the effect on ginsenosides of wild populations (correctively, $n=48)$ with the landrace population $(n=12)$, ginsenoside $\mathrm{Rb} 2$ was higher in wild populations than in landrace population in leaf ginsenosides, whereas ginsenosides

Table 1. Soil nutritional characteristics of wild and landrace (or cultivated) populations of american ginseng.

\begin{tabular}{|c|c|c|c|c|c|c|c|c|c|c|c|c|}
\hline \multirow[b]{2}{*}{ Population $^{z}$} & $\mathrm{~K}$ & $\mathrm{Ca}$ & $\mathrm{Mg}$ & $\mathrm{P}$ & $\mathrm{S}$ & $\mathrm{Fe}$ & $\mathrm{Mn}$ & $\mathrm{Zn}$ & $\mathrm{Cu}$ & $\mathrm{Al}$ & \multirow[b]{2}{*}{ Soil pH } & \multirow[b]{2}{*}{ OM $(\%)^{\mathrm{y}}$} \\
\hline & \multicolumn{10}{|c|}{$\left(\mathrm{mg} \cdot \mathrm{kg}^{-1}\right)$} & & \\
\hline P1 & $103.8^{x}$ & 3568.7 & 151.4 & 14.2 & 10.1 & 33.1 & 148.0 & 7.0 & 6.1 & 65.3 & 5.3 & 12.7 \\
\hline $\mathrm{P} 2$ & 95.5 & 526.9 & 58.0 & 9.49 & 8.7 & 81.3 & 65.8 & 3.9 & 2.7 & 186.1 & 4.6 & 7.4 \\
\hline P4 & 74.2 & 665.8 & 78.9 & 6.1 & 18.7 & 112.6 & 59.8 & 4.8 & 1.9 & 196.8 & 4.5 & 8.5 \\
\hline Mean $^{w}$ & 94.2 & 1557.2 & 95.8 & 8.6 & 11.7 & 77.3 & 83.0 & 5.1 & 2.9 & 146.0 & 4.8 & 10.1 \\
\hline P5 & 52.1 & 182.9 & 38.7 & 6.6 & 255.6 & 163.1 & 121.5 & 3.3 & 0.5 & 316.4 & 3.9 & 8.4 \\
\hline
\end{tabular}

${ }^{\mathrm{z}}$ Four wild populations (P1, P2, P3, and P4) were consecutively assigned based on finding order. Each wild population obtained from within Cornell University's Arnot Teaching and Research Forest (ATRF, Van Etten, NY) was physically separated from all other populations by at least $1.5 \mathrm{~km}$. P5 represents the landrace (or cultivated) population, which was cultivated at ATRF by using the seeds of cultivated ginseng landrace from Wisconsin.

${ }^{\mathrm{y}}$ Organic matter.

${ }^{\mathrm{x}}$ Values were pooled from 10 individual soil samples.

${ }^{\mathrm{w}}$ Mean value of four wild ginseng populations.

Table 2. Plant growth characteristics of four wild and one landrace populations in american ginseng.

\begin{tabular}{|c|c|c|c|c|c|c|c|c|c|}
\hline Population $^{z}$ & $\begin{array}{l}\text { Sympodium } \\
\text { length }(\mathrm{cm})\end{array}$ & $\begin{array}{c}\text { Leaf area } \\
\left(\mathrm{cm}^{2}\right)\end{array}$ & $\begin{array}{l}\text { Shoot } \\
\text { FW (g) }\end{array}$ & $\begin{array}{l}\text { Shoot } \\
\text { DW (g) }\end{array}$ & $\begin{array}{c}\text { Root } \\
\text { length }(\mathrm{cm})\end{array}$ & $\begin{array}{l}\text { Root diam } \\
(\mathrm{mm})\end{array}$ & $\begin{array}{c}\text { Root } \\
\text { FW (g) }\end{array}$ & $\begin{array}{c}\text { Root } \\
\text { DW (g) }\end{array}$ & $\begin{array}{c}\text { Root } \\
\text { age (yr) }\end{array}$ \\
\hline $\mathrm{P} 1$ & $18.7^{\mathrm{y}}$ & 183.0 & 3.1 & $1.0 \mathrm{a}^{\mathrm{x}}$ & 19.4 & $15.6 \mathrm{a}$ & $6.1 \mathrm{a}$ & $2.6 \mathrm{a}$ & $13.3 \mathrm{ab}$ \\
\hline $\mathrm{P} 2$ & 21.8 & 194.6 & 4.0 & $0.7 \mathrm{ab}$ & 22.0 & $13.6 \mathrm{ab}$ & $6.0 \mathrm{a}$ & $2.1 \mathrm{a}$ & $16.1 \mathrm{a}$ \\
\hline P4 & 16.7 & 166.4 & 4.1 & $0.7 \mathrm{ab}$ & 21.0 & $14.6 \mathrm{ab}$ & $7.1 \mathrm{a}$ & $2.3 \mathrm{a}$ & $12.9 \mathrm{ab}$ \\
\hline P5 & 18.4 & 174.1 & 3.4 & $0.5 \mathrm{~b}$ & 15.1 & $10.1 \mathrm{c}$ & $2.8 \mathrm{~b}$ & $0.9 \mathrm{~b}$ & $4.0 \mathrm{c}$ \\
\hline
\end{tabular}

${ }_{\mathrm{z}}^{\mathrm{z}}$ Four wild populations (P1, P2, P3, and P4) were consecutively assigned based on finding order. Each wild population obtained from within Cornell University's Arnot Teaching and Research Forest (ATRF, Van Etten, NY) was physically separated from all other populations by at least $1.5 \mathrm{~km}$. P5 represents the landrace population, which was cultivated at ATRF by using the seeds of landrace ginseng population from Wisconsin. ${ }^{\mathrm{y}}$ Values are the means of 12 replications per population $(n=12)$.

${ }^{\mathrm{x}}$ Means accompanied by the same letters are not significantly different in Duncan's multiple range tests at $P=0.05$.

$\mathrm{FW}=$ fresh weight; DW = dry weight. 
Table 3. The contents of individual and total ginsenosides from leaf and root organs of four wild and one landrace populations in american ginseng.

\begin{tabular}{|c|c|c|c|c|c|c|c|}
\hline \multirow[b]{2}{*}{ Population $^{\mathrm{z}}$} & \multicolumn{7}{|c|}{ Ginsenosides $\left(\mathrm{g} \cdot \mathrm{kg}^{-1} \mathrm{DW}\right)^{\mathrm{y}}$} \\
\hline & $\operatorname{Rg} 1$ & $\mathrm{Re}$ & $\mathrm{Rb} 1$ & $\mathrm{Rc}$ & $\mathrm{Rb} 2$ & $\mathrm{Rd}$ & Total \\
\hline \multicolumn{8}{|l|}{ Leaf } \\
\hline P1 & $2.5 \mathrm{c}^{\mathrm{x}}$ & $6.6 \mathrm{c}$ & 3.1 & $3.7 \mathrm{~d}$ & $10.6 \mathrm{~b}$ & $14.7 \mathrm{~b}$ & $41.1 \mathrm{~b}$ \\
\hline $\mathrm{P} 2$ & $4.1 \mathrm{ab}$ & $7.6 \mathrm{c}$ & 3.8 & $4.8 \mathrm{~b}$ & $10.4 \mathrm{~b}$ & $11.3 \mathrm{c}$ & $42.1 \mathrm{~b}$ \\
\hline P3 & $3.7 \mathrm{~b}$ & $7.4 \mathrm{c}$ & 4.0 & $4.3 \mathrm{c}$ & $7.7 \mathrm{c}$ & $10.5 \mathrm{c}$ & $37.5 \mathrm{~b}$ \\
\hline $\mathrm{P} 4$ & $4.4 \mathrm{a}$ & $9.5 \mathrm{~b}$ & 4.0 & $6.3 \mathrm{a}$ & $13.5 \mathrm{a}$ & $15.9 \mathrm{~b}$ & $53.5 \mathrm{a}$ \\
\hline P5 & $3.7 \mathrm{~b}$ & $12.0 \mathrm{a}$ & 4.7 & $4.0 \mathrm{~cd}$ & $7.4 \mathrm{c}$ & $22.7 \mathrm{a}$ & $54.4 \mathrm{a}$ \\
\hline \multicolumn{8}{|l|}{ Root } \\
\hline P1 & $15.9 \mathrm{a}$ & $0.0 \mathrm{~b}$ & $11.2 \mathrm{abc}$ & $2.9 \mathrm{~b}$ & 0.5 & $1.6 \mathrm{bc}$ & $32.1 \mathrm{abc}$ \\
\hline $\mathrm{P} 2$ & $14.2 \mathrm{a}$ & $0.0 \mathrm{~b}$ & $13.0 \mathrm{ab}$ & $3.8 \mathrm{a}$ & 0.5 & $1.5 \mathrm{bc}$ & $33.0 \mathrm{ab}$ \\
\hline P3 & $11.0 \mathrm{~b}$ & $0.0 \mathrm{~b}$ & $8.6 \mathrm{c}$ & $3.4 \mathrm{ab}$ & 0.5 & $1.0 \mathrm{c}$ & $24.4 \mathrm{c}$ \\
\hline $\mathrm{P} 4$ & $16.2 \mathrm{a}$ & $0.0 \mathrm{~b}$ & $14.4 \mathrm{a}$ & $3.2 \mathrm{ab}$ & 0.5 & $1.9 \mathrm{~b}$ & $16.2 \mathrm{a}$ \\
\hline P5 & $2.4 \mathrm{c}$ & $6.5 \mathrm{a}$ & $9.6 \mathrm{bc}$ & $3.6 \mathrm{ab}$ & 0.5 & $2.6 \mathrm{a}$ & $25.3 \mathrm{bc}$ \\
\hline \multicolumn{8}{|l|}{ Significance } \\
\hline Population (P) & NS & NS & NS & $*$ & $* * *$ & $*$ & $* *$ \\
\hline Organ $(\mathrm{O})$ & $* * *$ & $* * *$ & $* * *$ & $* *$ & $* * *$ & $* * *$ & $* * *$ \\
\hline Age (A) & $* *$ & NS & $* *$ & NS & NS & NS & $*$ \\
\hline $\mathrm{P} \times \mathrm{O}$ & $*$ & NS & NS & NS & $* * *$ & NS & NS \\
\hline $\mathrm{P} \times \mathrm{A}$ & NS & NS & NS & $*$ & NS & NS & $*$ \\
\hline $\mathrm{O} \times \mathrm{A}$ & $* * *$ & NS & $* *$ & $*$ & NS & NS & $* *$ \\
\hline $\mathrm{P} \times \mathrm{O} \times \mathrm{A}$ & NS & NS & NS & NS & NS & NS & NS \\
\hline
\end{tabular}

${ }^{\mathrm{z}}$ Four wild populations (P1, P2, P3, and P4) were consecutively assigned based on finding order. Each wild population obtained from within Cornell University's Arnot Teaching and Research Forest (ATRF, Van Etten, NY) was physically separated from all other populations by at least $1.5 \mathrm{~km}$. P5 represents the landrace population, which was cultivated at ATRF by using the seeds of cultivated ginseng landrace from Wisconsin. ${ }^{y}$ Values are the means of 12 replications per population $(n=12)$.

${ }^{x}$ Means accompanied by the same letters are not significantly different only within a column for leaf or root, respectively, in Duncan's multiple range tests at $P=0.05$.

Ns, * ${ }^{* *},{ }^{* * *}$ Not significant or significant at $P \leq 0.05,0.01$, or 0.001 , respectively.

$\mathrm{Re}, \mathrm{Rd}$, and total ginsenoside were greater in the landrace population. By contrast, ginsenosides $\mathrm{Rg} 1, \mathrm{Rb} 1$, and $\mathrm{Rc}$ did not show any difference between wild and the landrace population. On the other hand, root ginsenoside Rg1, and total ginsenoside were greater in wild populations than in landrace population, whereas root ginsenoside Re was higher in landrace population than in wild populations. Of individual ginsenosides, ginsenoside Rg1 was the highest in root from overall populations, whereas ginsenoside $\mathrm{Rd}$ was the greatest in leaves from all populations.

The effect of the organ difference on the compositions and contents of ginsenosides within each population was evaluated. The content and composition of ginsenosides from wild and landrace populations, respectively, were significantly different between leaf and root organs. Within the four wild populations, ginsenosides $\mathrm{Rg} 1$ and $\mathrm{Rb} 1$ were greater in root than in leaf, whereas ginsenoside $\mathrm{Rb} 1$ was higher in the root than in the leaf only within the landrace population. Ginsenoside $\mathrm{Re}, \mathrm{Rb} 2$, and $\mathrm{Rd}$ were greater in leaves than in roots for both wild and landrace populations. The contents of total ginsenoside from both wild and landrace populations were significantly greater in leaf than in root tissues. In a 4-year-old landrace population, total ginsenoside content was $\approx 2$-fold higher in leaf than in root.

As the contents of leaf and root ginsenosides were determined, it became pertinent to test whether leaf ginsenosides were associated with accumulation of root ginsenosides (Table 4). No significant effect of leaf ginsenosides on chemotypic responses of root ginsenosides was found, but the effects of population and
Table 4. Significance levels for effects of population, covariate leaf ginsenoside, and interactions on the contents of individual and total root ginsenosides from four wild and one landrace populations of american ginseng.

\begin{tabular}{lccccccc}
\hline & \multicolumn{7}{c}{ Root ginsenosides } \\
\cline { 2 - 7 } Factors & Rg1 & Re & Rb1 & Rc & Rb2 & Rd & Total \\
\hline Population (P) & NS & NS & NS & NS & NS & $* * *$ & NS \\
Leaf ginsenosides (LG) & NS & NS & NS & NS & NS & NS & NS \\
$P \times$ LG & NS & NS & NS & NS & NS & $* *$ & NS \\
\hline
\end{tabular}

$\overline{\text { ss, }{ }^{* *},{ }^{* * *} \text { Not significant or significant at } P \leq 0.01 \text { or } 0.001 \text {, respectively. }}$

interaction between population and leaf ginsenoside variables were detected only in the case of ginsenoside Rd (Table 4).

Polynomial regression analyses were performed between total ginsenoside contents of leaf/root and the estimated ginseng storage root age as independent variables, only from wild populations (Fig. 1). The estimated ginseng root age was negatively correlated with total leaf ginsenoside but only in population $1[P=0.038$ (Fig. 1A)], whereas no quadratic or cubic regression effect appeared in population 1 . However, two positive relationships between estimated ginseng root age and total root ginsenosides were detected in wild populations 2 and $4[P=0.004$ and $P=0.005$, respectively (Fig. 1B)]. Linear, quadratic and cubic regression analyses were detected in population 2, whereas linear and quadratic regression responses only appeared in population 4 .

To evaluate the relationship among plant growth parameters, individual and total ginsenosides between root and foliar 

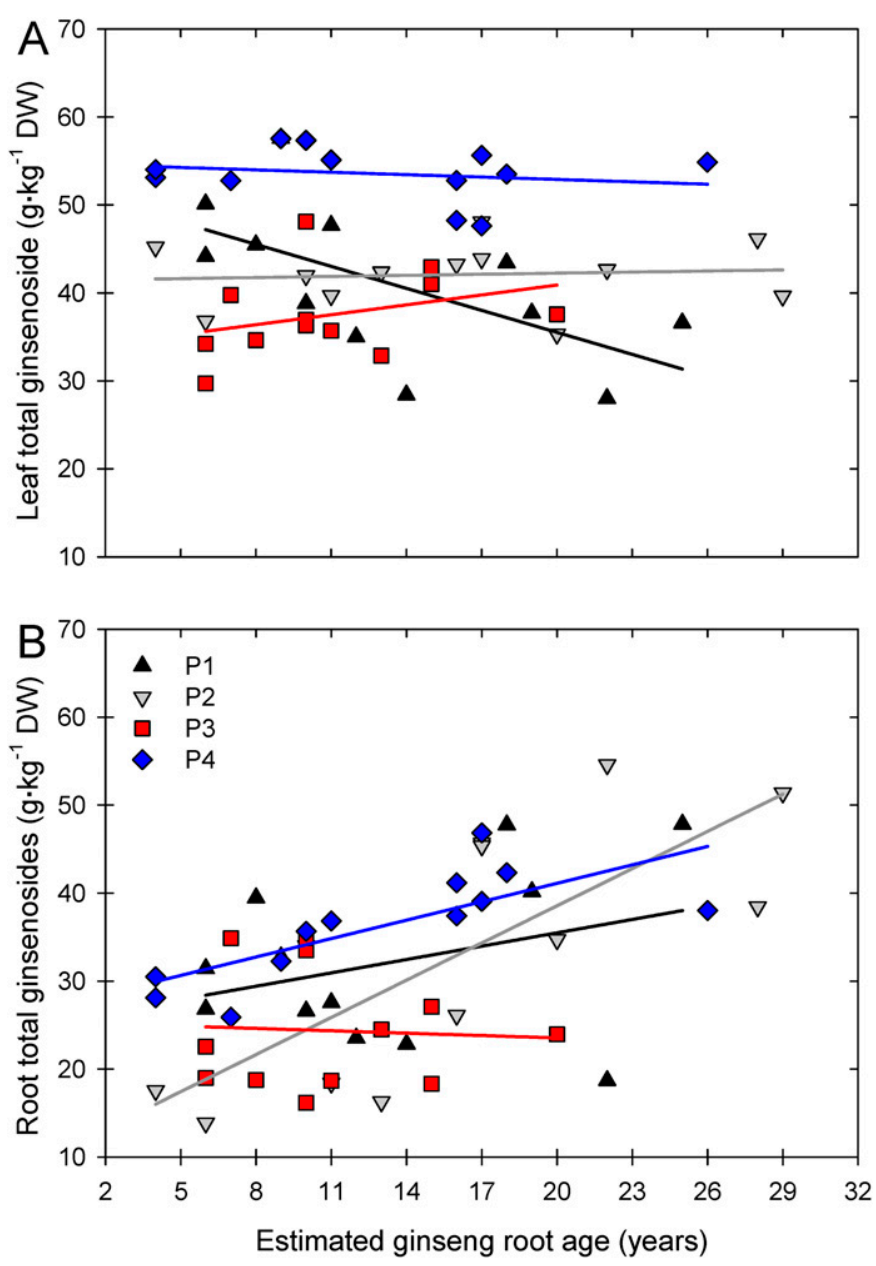

Fig. 1. Relationship between estimated storage root age and total ginsenoside contents from leaf (A) and root (B) organs of four wild populations in american ginseng $(n=12)$. Regression equations for total leaf ginsenoside (A) are $y=-0.834 x+52.192\left(r^{2}=0.364, P=0.038\right)$ for population $1(\mathrm{P} 1$, black line), $y=0.041 x+41.420\left(r^{2}=0.008, P=0.787\right)$ for population $2(\mathrm{P} 2$, gray line), $y=0.376 x+33.383\left(r^{2}=0.103, P=0.308\right)$ for population $3(\mathrm{P} 3$, red line), and $y=-0.091 x+54.716\left(r^{2}=0.037, P=0.547\right)$ for population $4(\mathrm{P} 4$, blue line). Regression equations for total root ginsenoside (B) are $y=0.506 x+$ $0.506\left(r^{2}=0.109, P=0.295\right)$ for population 1 (P1, black line), $y=1.409 x+$ $10.379\left(r^{2}=0.589, P=0.004\right)$ for population $2(\mathrm{P} 2$, gray line $), y=-0.090 x+$ $25.351\left(r^{2}=0.003, P=0.863\right)$ for population $3(\mathrm{P} 3$, red line $)$, and $y=0.698 x+$ $27.157\left(r^{2}=0.563, P=0.005\right)$ for population $4(\mathrm{P} 4$, blue line $)$. Four wild populations ( $\mathrm{P} 1, \mathrm{P} 2, \mathrm{P} 3$, and $\mathrm{P} 4)$ were consecutively assigned based on finding order. Each wild population obtained from within Cornell University's Arnot Teaching and Research Forest in Van Etten, NY was physically separated from all other populations by at least $1.5 \mathrm{~km}$. DW = dry weight.

organs, a Pearson correlation analysis was performed based on the responses of plant growth characteristics and the contents of individual and total ginsenosides between the two organs from wild and landrace populations, respectively (Fig. 2). In wild populations, plant growth characteristics except for leaflet number were positively correlated with each other. With the exception of ginsenoside $\mathrm{Re}$, root ginsenosides were positively correlated with each other. Ginsenoside Re was not detected in wild populations. However, most leaf ginsenosides were positively correlated with each other but some leaf ginsenosides, such as Rb1 and Rg1 were negatively correlated (Fig. 2A). On the other hand, in the landrace population, plant FW and DW were strongly positively correlated with each other but certain plant growth characteristics, such as leaf number, plant height, root length, and root diameter, were negatively correlated with each other. Root age did not correspond to the other parameters because root age in landrace population was identical as those seeds were seeded and harvested at the same date. Most root ginsenosides were positively correlated except that root ginsenoside $\mathrm{Rg} 1$ was negatively correlated with other root ginsenosides. By contrast, leaf ginsenosides were differently associated with each other (Fig. 2B).

It is most likely that plant growth characteristics more highly positively correlated with root and leaf ginsenosides in landrace population than in wild populations. Especially, leaf ginsenoside $\mathrm{Rd}$ and leaf total ginsenoside were positively correlated with plant height, shoot FW, LAI, and shoot and leaf DW. However, the positive correlation between root and leaf ginsenosides was much stronger in the wild population than in the landrace population. That is, root ginsenosides $\mathrm{Rg} 1, \mathrm{Rb} 1$, $\mathrm{Rd}$, and total ginsenoside were positively correlated with leaf ginsenosides except for leaf ginsenosides $\mathrm{Rg} 1$ and $\mathrm{Rb} 1$ in wild populations. In the landrace population, there were only two significant correlations between leaf and root ginsenosides; leaf ginsenoside $\mathrm{Rg} 1$ was positively and negatively correlated with root ginsenoside $\mathrm{Rg} 1$ and $\mathrm{Re}$, respectively.

Principal component analysis models were performed to provide the overall responses of all the ginsenosides to the given ginseng populations, depending on organ types. For leaf ginsenoside, principal components 1 (PC-1) and 2 (PC-2) accounted for $44 \%$ and $17 \%$ of variance, respectively (Fig. $3 \mathrm{~A})$. For root ginsenoside, PC-1 and PC-2 accounted for $51 \%$ and $25 \%$ of variance, respectively (Fig. 3B). In both of PCA scores plots, the landrace population was clearly separated from all wild populations. However, ginsenosides were more closely linked with each other in leaves (Fig. 3A) than in roots (Fig. 3B).

Hierarchical cluster analysis was also performed to evaluate the similarities among wild and landrace populations based on the results of individual and total ginsenosides contents. Two clusters (clusters A and B vs. clusters C and D) were identified in terms of organ types, root and leaf (Fig. 4). Within each organ, two clusters were observed. In leaves, populations 1,2 , and 3 as the first cluster (cluster B) were most tightly located, whereas populations 4 and 5 belonged to the second cluster (cluster A). In roots, populations 1, 2, and 4 as the first cluster (cluster D) were most closely clustered, whereas populations 3 and 5 as the second cluster (cluster C) were grouped.

\section{Discussion}

The content and composition of individual ginsenosides in american ginseng are affected by both environmental cues and genotypic variations (Lim et al., 2005, 2006; Mudge et al., 2004). In previous work, the metabolic diversity of ginsenoside contents and compositions derived from genotypic variations was mainly considered, based on a geographically broad range as a sampling approach (Assinewe et al., 2003; Dong et al., 2003; Jackson et al., 2003; Mudge et al., 2004; Schlag and McIntosh, 2006). However, high ginsenoside variation was apparent within small populations, such as a single farm unit (Hong et al., 2005). These studies suggest that chemotypic diversity of ginsenoside profiles might occur within a small scale with high resolution regarding a natural diversity of ginseng populations. Therefore, we screened four wild 


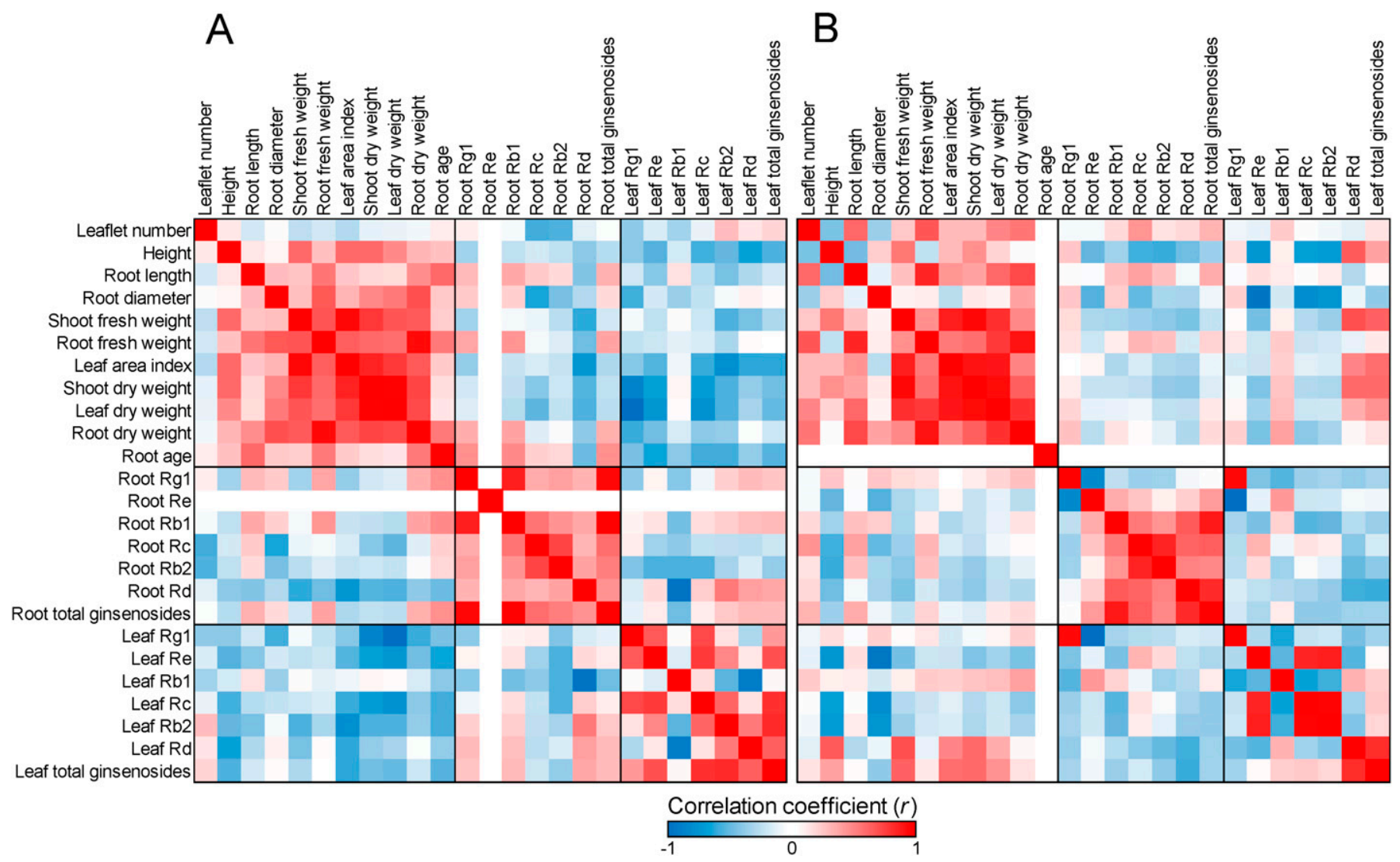

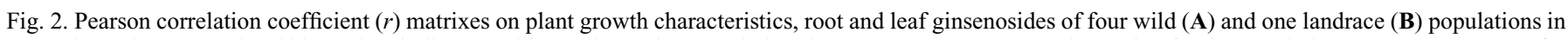
american ginseng. Red and blue colors indicate positive and negative correlations between parameters. Sample number for the correlation test was $48(n=48)$ for four wild populations and $12(n=12)$ for one landrace population.

american ginseng populations separated by only a relatively short physical distance to test the hypothesis that the chemotypic variation of ginsenoside contents and compositions might be detected in wild populations with a small scale range.

Although we used wild ginseng populations with a small scale range, it appeared that the compositions and contents of leaf and root ginsenosides were considerably different among all wild populations and between wild and landrace populations. In wild populations, roots had the highest contents of ginsenosides $\mathrm{Rg} 1$ and $\mathrm{Rb} 1$. The results are in agreement with studies that found that $\mathrm{Rg} 1$ was the most abundant ginsenoside in roots, even within a broad (low resolution) sampling area (Assinewe et al., 2003; Lim, 2005; Lim et al., 2005; Schlag and McIntosh, 2006). By contrast, root ginsenoside Re was not detected in wild populations in this study, whereas root ginsenoside Re in previous work was broadly distributed within more widely distributed wild populations (Lim et al., 2005; Schlag and McIntosh, 2006). This difference might be derived from the reason that the wild populations in this study would be more isolated than the wild populations in the studies of Lim et al. (2005) and Schlag and McIntosh (2006), based on the different response patterns of root ginsenosides Re and Rg1. Also, it is assumed that these inverse responses of ginsenosides $\mathrm{Re}$ and $\mathrm{Rg} 1$ might result from the absence, downregulation of ginsenoside Re or both, compared with ginsenoside $\mathrm{Rg} 1$. On the other hand, there might be a competition on the accumulation of ginsenosides Rg1 and Re because both ginsenosides share the same biosynthetic pathway by presumable upregulation of cytochrome $\mathrm{P} 450$ and glycosyltransferase, followed by biosynthesis of protopanaxadiol (Kim et al., 2006; Liang and Zhao, 2008). This result suggests that root ginsenoside Re might be used as a phytochemical biomarker for detecting the certain authentic wild american ginseng population, as opposed to introduced populations (e.g., wild simulated plantings in unmanaged forest settings). Moreover, natural american ginseng populations would be conserved in terms of the absence of root ginsenoside Re from wild populations with a small scale range. Also, based on the results of the PCA models, these wild populations were clearly distinguished from landrace population. Furthermore, the relative abundance of individual ginsenosides is similar in wild and landrace populations (Assinewe et al., 2003; Li et al., 1996; Lim, 2005; Lim et al., 2005). The content of total root ginsenosides was $\approx 30 \mathrm{~g} \cdot \mathrm{kg}^{-1}(3 \%) \mathrm{DW}$ in both wild and landrace populations, which is consistent with the other results (Li and Mazza, 1999; Li et al., 1996; Lim et al., 2005). However, Assinewe et al. (2003) reported that total root ginsenoside from wild ginseng populations was $5.8 \%$, which was $\approx 2-$ fold higher than the results presented here.

The composition (relative abundance) of individual leaf ginsenoside wild and landrace populations were different. Leaf ginsenosides $\mathrm{Re}$ and $\mathrm{Rd}$ were greater in landrace population than in wild populations. The difference of these two leaf ginsenosides could contribute to the higher accumulation of total leaf ginsenosides in landrace population. The relative abundance of leaf ginsenosides in this study is consistent with the result of Assinewe et al. (2003). That is, leaf ginsenosides 

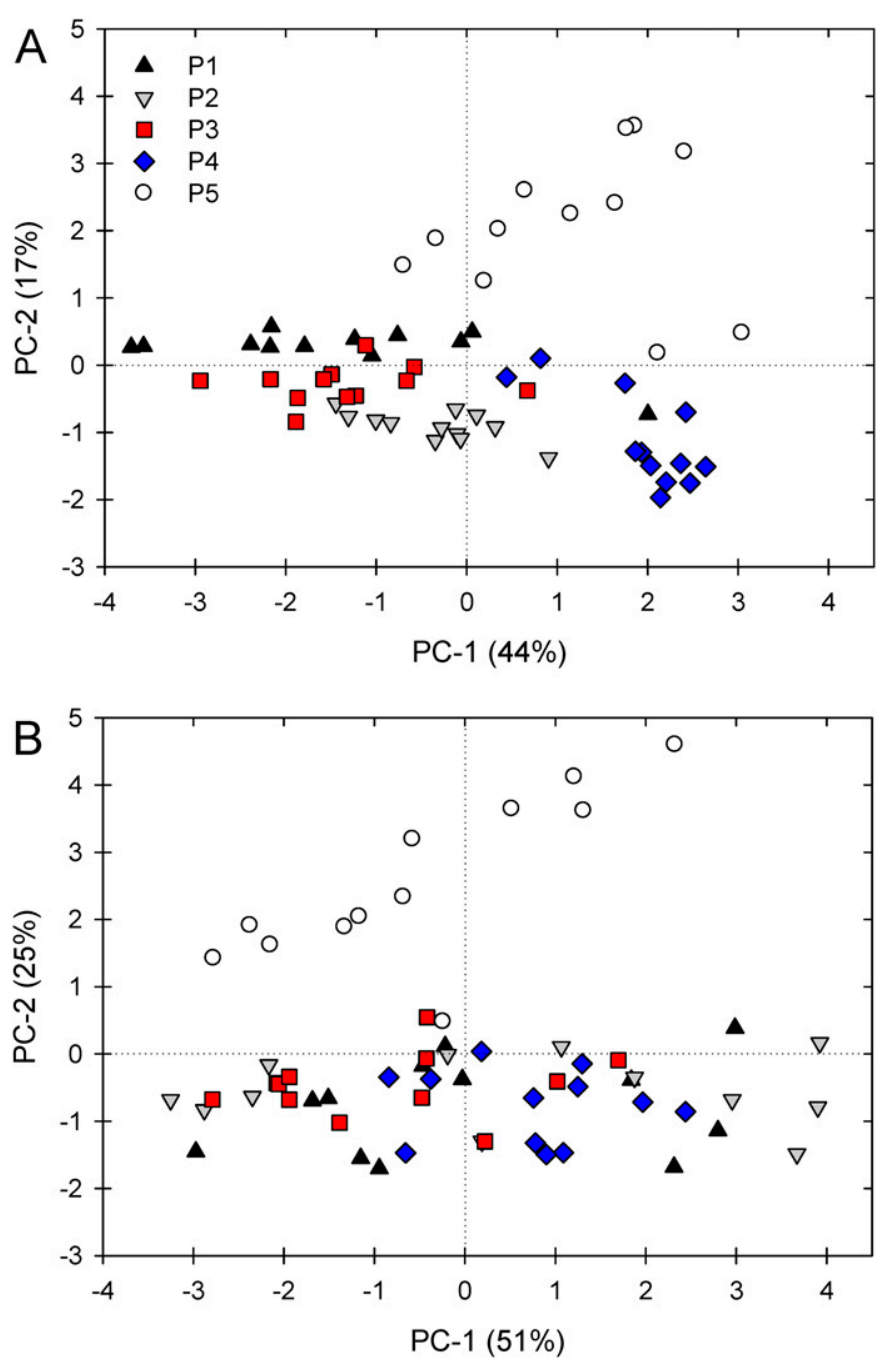

Fig. 3. Two-dimensional scores plots of the principal component (PC) analysis of leaf (A) and root (B) ginsenosides from four wild and one landrace populations in american ginseng. Each population represents the mean of 12 replications $(n=12)$.

$\mathrm{Re}, \mathrm{Rb} 2$, and $\mathrm{Rd}$ had a higher content than leaf ginsenosides $\mathrm{Rg} 1, \mathrm{Rb} 1$, and Rc. In this study, total leaf ginsenosides in both wild and landrace populations was $\approx 50 \mathrm{~g} \cdot \mathrm{kg}^{-1}(5 \%)$ on the basis of DW. This result is consistent with that of Li and Mazza (1999) and Li et al. (1996), although Assinewe et al. (2003) reported that leaf ginsenoside was only $3.3 \%$. It is assumed that the difference in leaf ginsenoside contents between wild and landrace populations could be related to different environmental factors, including soil nutrient and soil moisture content.

Alongside the genotypic variation of ginsenoside compositions among wild populations having a high resolution, the organ difference on the compositions and contents of leaf and root ginsenosides was also observed within wild and landrace populations, respectively. That is, the relative abundance of leaf and root ginsenosides showed different response patterns among the different wild populations. The relative abundance of leaf ginsenosides but not of root ginsenosides in this study was fairly consistent with that from the 4-year-old wild american ginseng reported by Assinewe et al. (2003). This difference might be due to reduction, absence of ginsenoside Re in this study or both, compared with the results of Assinewe et al. (2003). This response in root ginsenoside $\mathrm{Re}$ is also reported by Lim et al. (2005), who reported the inverse relationship between $\mathrm{Re}$ and $\mathrm{Rg} 1$ from eight wild populations. Furthermore, the ratio of root ginsenoside $\mathrm{Rg} 1$ and $\mathrm{Re}$ was highly diverse within wild or landrace populations (Schlag and McIntosh, 2006). In this study, only root ginsenoside Rg1 but not root ginsenoside $\mathrm{Re}$ was detected. In addition, to the accumulation of ginsenosides from wild populations, the most abundant leaf ginsenosides from landrace population were $\mathrm{Rd}$, $\mathrm{Re}$, and $\mathrm{Rb} 2$ in that order, consistent with the results of $\mathrm{Li}$ et al. (1996), and Li and Mazza (1999). In the case of the least abundant leaf ginsenosides, there was less variation in ginsenosides Rg1, Rb1, and Rc, compared with other studies ( $\mathrm{Li}$ and Mazza, 1999; Li et al., 1996). Furthermore, Li and Wardle (2002) reported that the two most abundant leaf ginsenosides were ginsenosides $\mathrm{Rd}$ and $\mathrm{Re}$ but the two least abundant ginsenosides were Rbl and Rc. In this study, the three most abundant root ginsenosides in landrace population were Rb1, $\mathrm{Re}$, and $\mathrm{Rd}$. The result was similar to the previous reports ( $\mathrm{Li}$ and Mazza, 1999; Li and Wardle, 2002; Li et al., 1996). The content of total leaf ginsenoside was higher than that of total root ginsenosides in a 6-year-old asian ginseng (Kim and Choi, 1987).

We evaluated the effect of the storage root age on the contents of total ginsenosides in both leaf and root. Total leaf ginsenosides did not show any significant positive correlation with estimated storage root age, whereas total root ginsenosides was positively correlated with estimated storage root age in two of four wild populations (Fig. 1). Mudge et al. (2004) observed that root ginsenoside $\mathrm{Rb} 1$ from six of eight wild populations increased with the storage root age. Furthermore, Shi et al. (2007) and Qu et al. (2009) reported that the contents of total root ginsenosides increased with increase in a storage root age. However, as in this result, the contents of total leaf ginsenosides were unaffected by the estimated ginseng root age $(\mathrm{Qu}$ et al., 2009; Shi et al., 2007). It is likely that the composition and content of leaf ginsenosides in wild populations might be more affected by adverse environmental stimuli, including soil nutrient and light levels in the understory of deciduous forests than genotypic factors (Fournier et al., 2003; Konsler et al., 1990). Even in the ANOVA results, age was less influenced by the ginsenoside variables than the organ factor (Table 2). Considering the organ factor for the interaction effect, the age factor was less significant in the contents of individual and total ginsenosides than organ factor because the main effect on age was only detected in ginsenosides $\mathrm{Rg} 1, \mathrm{Rb} 1$, and total ginsenosides, whereas all the individual and total ginsenosides were significantly influenced by organ factor. Furthermore, leaf ginsenosides did not influence accumulation of root ginsenosides based on the result of the ANCOVA, in which leaf ginsenosides were analyzed as a covariant (Table 3 ). These results indicate that leaf ginsenosides may accumulate independently of root ginsenosides. Thus, it is highly considered that the contents of leaf ginsenosides might be more affected by abiotic factors than by genotypic factors. Nevertheless, the higher abundance of leaf ginsenoside (Qu et al., 2009; Shi et al., 2007; Starratt et al., 2001; Wang et al., 2006) might make it medicinally useful (Xie et al., 2004), although ginseng leaf has been considerably less used for the commercial application than ginseng root.

The significance of the main and all interaction effects on ginsenoside accumulation, shown in Table 3 , indicates that the 


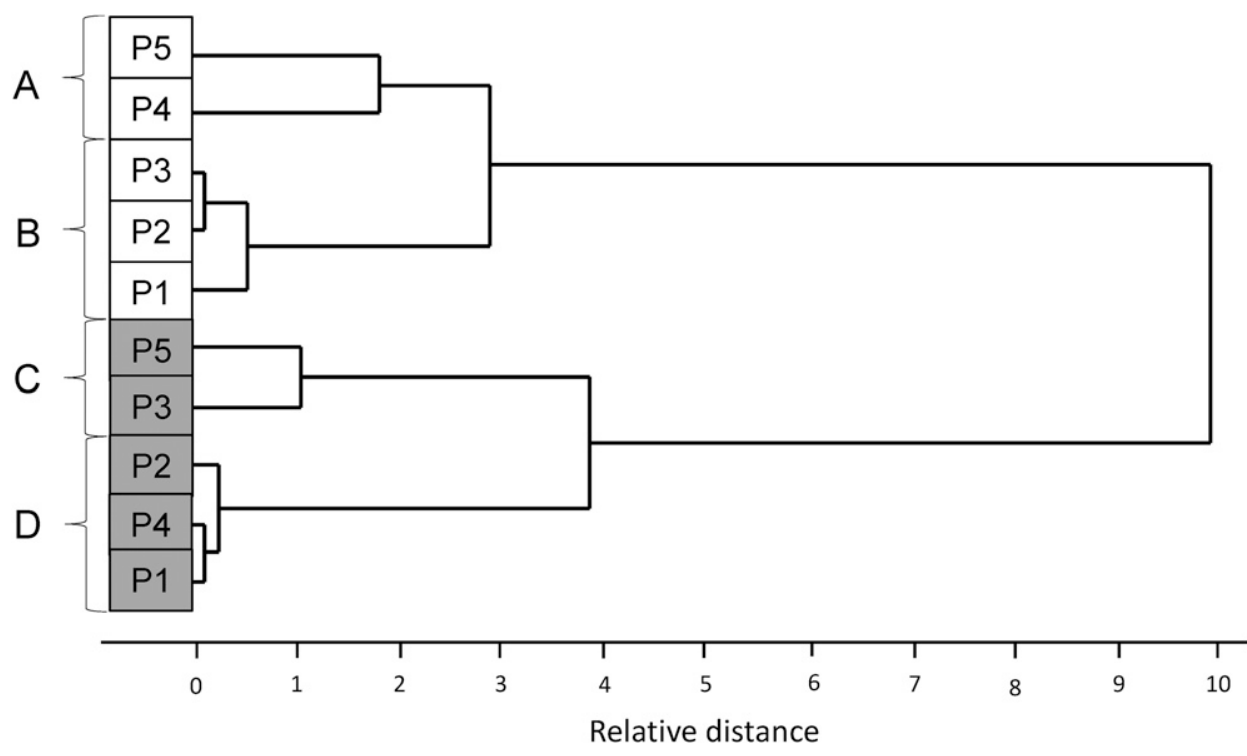

Fig. 4. Dendogram of hierarchical cluster analysis (HCA) using hierarchical complete linkage clustering method of leaf and root ginsenosides from four (P1, P2, P3, and P4) wild and one (P5) landrace populations in american ginseng. In HCA, clustering of samples is based on their similarity. Each component (each population) represents the mean value of 12 samples $(n=12)$. White and gray boxes indicate root and leaf, respectively. Four wild populations (P1, P2, P3, and P4) were consecutively assigned based on finding order. Each wild population obtained from within Cornell University's Arnot Teaching and Research Forest (ATRF, Van Etten, NY) was physically separated from all other populations by at least $1.5 \mathrm{~km}$. P5 represents the landrace ginseng population that was cultivated at ATRF by using the seeds of cultivated ginseng landrace from Wisconsin.

indicates that certain individual leaf ginsenosides could be used as indicators for the estimation of individual, total root ginsenosides without destructive root sampling or both.

In conclusion, there was considerable genotypic variation in the compositions and contents of ginsenosides between leaf and root from wild and landrace populations. The relative abundance of individual ginsenosides between leaf and root differed between wild and landrace populations. Total ginsenoside was higher in leaf than in root from both wild and landrace populations. Storage root age was not a good predictor of total leaf ginsenosides but it was positively correlated with total root ginsenosides in certain wild populations. Also, there were certainly the main and two-way interaction effects on all individual ginsenosides and total ginsenosides, whereas there was no three-way interaction effect. The correlation analysis revealed that leaf ginsenosides Rd and Rg1 had moderate and

main effects of population and organ were similar except for the significance of ginsenosides $\operatorname{Rg} 1$ and $\mathrm{Re}$ on the organ factor, whereas the main effects of population and age differed considerably. All two-way interaction effects on population $\times$ organ, population $\times$ age, and organ $\times$ age were different from each other for accumulation of ginsenosides. Lim et al. (2005) found that the effects of population and age were considerably different at 0-2 years after transplanting. By contrast, root age effect was not statistically detected at 1 year after transplanting, whereas only the population effect appeared in all individual and total ginsenosides (Lim, 2005). The interaction effects of population and age were apparent with all ginsenosides, except for ginsenoside $\operatorname{Re}$ (Lim, 2005) but in this study, we observed the interaction effect of populations and age on ginsenoside Rc and total ginsenosides.

To test the hypothesis that a certain ginsenoside in leaf might be related with the corresponding, other ginsenosides accumulation in root from the wild and cultivated ginseng populations or both, respectively, we performed the Pearson's correlation procedure on both leaf and root ginsenosides to predict root ginsenoside contents from the estimation of leaf ginsenoside levels. In wild populations, within all possible correlation combinations, the most significant combination was root ginsenoside $\mathrm{Rd}$ with leaf ginsenoside $\mathrm{Rb} 2$. On the other hand, in landrace population, only two combinations showed the significant correlation of leaf ginsenoside $\mathrm{Rg} 1$ with root ginsenosides $\operatorname{Rg} 1(r=0.943, P<0.0001)$ and $\operatorname{Re}(r=-0.766$, $P=0.004)$, respectively (Fig. $2 \mathrm{~B})$. This result is consistent with the result of $\mathrm{Li}$ et al. (1996), revealing that the correlations between leaf and root ginsenosides from nine cultivated ginseng populations were very low. In the 4-year-old american ginseng, total root ginsenoside was positively correlated with leaf ginsenosides Rd, Re, and Rb2 (Konsler et al., 1990). This strong correlation with root ginsenosides $\mathrm{Rb} 2$ and $\mathrm{Rg} 1$ from wild and landrace populations, respectively. Furthermore, PCA and cluster analysis revealed that these wild populations differed from the landrace ginseng population. Leaf ginsenosides might not be affected by the estimated root age. Nevertheless, certain leaf ginsensoide might be useful as a potential phytochemical biomarker to estimate root ginsenosides in commercial settings, to screen the wild ginseng populations, and to select landrace populations for higher yield of ginsenosides.

\section{Literature Cited}

An, Y.N., S.Y. Lee, M.G. Choung, and K.H. Kang. 2002. Ginsenoside concentration and chemical component as affected by harvesting time of four-year ginseng root. Korean J. Crop Sci. 47:216-220.

Assinewe, V., B.R. Baum, D. Gagnon, and J.T. Arnason. 2003. Phytochemistry of wild populations of Panax quinquefolius L. (north american ginseng). J. Agr. Food Chem. 51:4549-4553.

Attele, A.S., J.A. Wua, and C-S. Yuan. 1999. Ginseng pharmacology: Multiple constituents and multiple actions. Biochem. Pharmacol. 58:1685-1693.

Chuang, W.C. and S.J. Sheu. 1994. Determination of ginsenosides in ginseng crude extracts by high-performance liquid chromatography. J. Chromatography 685:243-251.

Chung, I-M., J-W. Kim, P. Seguin, Y-M. Jun, and S-H. Kim. 2012. Ginsenosides and phenolics in fresh and processed korean ginseng (Panax ginseng C.A. Meyer): Effects of cultivation location, year, and storage period. Food Chem. 130:73-83.

Corbit, R.M., J.F.S. Ferreira, S.D. Ebbs, and L.L. Murphy. 2005. Simplified extraction of ginsenosides from american ginseng (Panax quinquefolius L.) for high-performance liquid chromatographyultraviolet analysis. J. Agr. Food Chem. 53:9867-9873.

Court, W.A., L.B. Reynolds, and J.G. Hendel. 1996. Influence of root age on the concentration of ginsenosides of american ginseng (Panax quinquefolium). Can. J. Plant Sci. 76:853-855. 
Dong, T.T.X., X.M. Cui, Z.H. Song, K.J. Zhao, Z.N. Ji, C.K. Lo, and K.W.K. Tsim. 2003. Chemical assessment of roots of Panax notoginseng in China: Regional and seasonal variations in its active constituents. J. Agr. Food Chem. 51:4617-4623.

Fournier, A.R., J.T.A. Proctor, L. Gauthier, S. Khanizadeh, A. Belanger, A. Gosselin, and M. Dorais. 2003. Understory light and root ginsenosides in forest-grown Panax quinquefolius. Phytochemistry $63: 777-782$.

Hong, D.Y.Q., A.J. Lau, C.L. Yeo, X.K. Liu, C.R. Yang, H.L. Koh, and Y. Hong. 2005. Genetic diversity and variation of saponin contents in Panax notoginseng roots from a single farm. J. Agr. Food Chem. 53:8460-8467.

Jackson, C.J.C., J.P. Dini, C. Lavandier, H. Faulkner, H.P.V. Rupasinghe, and J.T.A. Proctor. 2003. Ginsenoside content of north american ginseng (Panax quinquefolius L. Ararliaceae) in relation to plant development and growing locations. J. Ginseng Res. 27:135-140.

Jochum, G.M., K.W. Mudge, and R.B. Thomas. 2007. Elevated temperatures increase leaf senescence and root secondary metabolite concentrations in the understory herb Panax quinquefolius (Araliaceae). Amer. J. Bot. 94:819-826.

Kim, S-C. and K-J. Choi. 1987. Content comparison of proximate compositions, various solvent extracts and saponins in root, leaf and stem of Panax ginseng. Korean J. Ginseng Sci. 11:118-122.

Kim, M.K., B-S. Lee, J-G. In, H. Sun, J-H. Yoon, and D-C. Yang. 2006. Comparative analysis of expressed sequence of tags (ESTs) of ginseng leaf. Plant Cell Rpt. 25:599-606.

Kim, S.K., I. Sakamoto, K. Morimoto, and M. Sakata. 1981. Seasonal variation of saponins, sucrose and monosaccharides in cultivated ginseng roots. Planta Med. 42:181-186.

Konsler, T.R. and J.E. Shelton. 1990. Lime and phosphorus effects on american ginseng: I. Growth, soil fertility, and root tissue nutrient status response. J. Amer. Soc. Hort. Sci. 115:570-574.

Konsler, T.R., S.W. Zito, J.E. Shelton, and E.J. Staba. 1990. Lime and phosphorus effects on american ginseng: II. Root and leaf ginsenoside content and their relationship. J. Amer. Soc. Hort. Sci. 115:575580 .

Lee, M-J., J-S. Choi, S-W. Cha, K-S. Lee, Z-W. Lee, G-S. Hwang, S.H. Lee, A.H.M. Kamal, Y-A. Jung, N-S. Seung, and S-H. Woo. 2011. Variation in the ginsenoside profiles of cultivated ginseng (Panax ginseng C.A. Meyer) landraces in Korea. Process Biochem. 46:258264.

Lee, J. and K.W. Mudge. 2013a. Gypsum effects on plant growth, nutrients, ginsenosides, and their relationship in american ginseng. Hort. Environ. Biotechnol. 54:228-235.

Lee, J. and K.W. Mudge. 2013b. Water deficit affects plant and soil water status, plant growth, and ginsenoside contents in american ginseng. Hort. Environ. Biotechnol. 54:475-483.

Li, T.S.C. 1995. Asian and american ginseng-A review. HortTechnology 5:27-34.

Li, T.S.C. and G. Mazza. 1999. Correlations between leaf and soil mineral concentrations and ginsenoside contents in american ginseng. HortScience 34:85-87.

Li, T.S.C., G. Mazza, A.C. Cottrell, and L. Gao. 1996. Ginsenosides in roots and leaves of american ginseng. J. Agr. Food Chem. 44:717720 .

Li, T.S.C. and D. Wardle. 2002. Seasonal fluctuations of leaf and root weight and ginsenoside contents of 2-, 3-, and 4-year-old american ginseng plants. HortTechnology 12:229-231.
Liang, Y. and S. Zhao. 2008. Progress in understanding of ginsenoside biosynthesis. Plant Biol. 10:415-421.

Lim, W. 2005. Effects of interaction among age, cultivation method (location), and population on ginsenoside content of wild Panax quinquefolium L., one year after transplanting from wild. Korean J. Med. Crop Sci. 13:254-261.

Lim, W., K.W. Mudge, and J. Lee. 2006. Effect of water stress on ginsenoside production and growth of american ginseng. HortTechnology 16:517-522.

Lim, W., K.W. Mudge, and F. Vermeylen. 2005. Effects of population, age, and cultivation methods on ginsenoside content of american ginseng (Panax quinquefolium). J. Agr. Food Chem. 53:8498-8505.

Luchtefeld, R., E. Kostoryz, and R.E. Smith. 2004. Determination of ginsenosides Rb1, Rc, and Re in different dosage forms of ginseng by negative ion electrospray liquid chromatography-mass spectrometry. J. Agr. Food Chem. 52:4953-4956.

Mahady, G.B., C. Gyllenhaal, H.H.S. Fong, and N.R. Farnsworth. 2000. Ginsengs: A review of safety and efficacy. Nutr. Clin. Care 3:90-101.

Mudge, K.W., W. Lim, J.P. Lardner, and R.L. Beyfuss. 2004. Effects of population and age on ginsenoside content of american ginseng (Panax quinquefolium L.). Acta Hort. 629:161-166.

Nam, K.Y., S.R. Ko, and K.J. Choi. 1998. Relationship of saponin and non-saponin for the quality of ginseng. J. Ginseng Res. 22:274-283.

Proctor, J.T.A. and W.G. Bailey. 1987. Ginseng: Industry, botany, and culture. Hort. Rev. 9:187-236.

Qi, L.W., C.Z. Wang, and C.S. Yuan. 2011. Ginsenosides from american ginseng: Chemical and pharmacological diversity. Phytochemistry 72:689-699.

Qu, C., Y. Bai, X. Jin, Y. Wang, K. Zhang, J. You, and H. Zhang. 2009. Study on ginsenosides in different parts and ages of Panax quinquefolius L. Food Chem. 115:340-346.

Schlag, E.M. and M.S. McIntosh. 2006. Ginsenoside content and variation among and within american ginseng (Panax quinquefolius L.) populations. Phytochemistry 67:1510-1519.

Shi, W., Y.T. Wang, J. Li, H.Q. Zhang, and L. Ding. 2007. Investigation of ginsenosides in different parts and ages of Panax ginseng. Food Chem. 102:664-668.

Sivakumar, G., K.W. Yu, and K.Y. Paek. 2005. Biosafe ginseng: A novel source for human well-being. Eng. Life Sci. 5:527-533.

Smith, R.G., D. Caswell, A. Carriere, and B. Zielke. 1996. Variation in the ginsenoside content of american ginseng, Panax quinquefolius $\mathrm{L}$. roots. Can. J. Bot. 74:1616-1620.

Soldati, F. and O. Tanaka. 1984. Panax ginseng: Relation between age of plant and content of ginsenosides. Planta Med. 50:351-352.

Starratt, A.N., J.G. Hendel, and R.D. Reeleder. 2001. Leaves of north american ginseng, Panax quinquefolius L.: A renewable source of certain ginsenosides. Can. J. Plant Sci. 81:65-67.

Wang, A., C-Z. Wang, J-A. Wu, J. Osinski, and C-S. Yuan. 2005. Determination of major ginsenosides in Panax quinquefolius (american ginseng) using high-performance liquid chromatography. Phytochem. Anal. 16:272-277.

Wang, C-Z., J.A. Wu, E. McEntee, and C-S. Yuan. 2006. Saponins composition in american ginseng leaf and berry assayed by highperformance liquid chromatography. J. Agr. Food Chem. 54:22612266.

Xie, J-T., S.R. Mehendale, A. Wang, A.H. Han, J.A. Wu, J. Osinski, and C-S. Yuan. 2004. American ginseng leaf: Ginsenoside analysis and hypoglycemic activity. Pharmacol. Res. 49:113-117. 